EPJ Web of Conferences 41, 10008 (2013)

DOI: $10.1051 /$ epjconf/20134110008

C) Owned by the authors, published by EDP Sciences, 2013

\title{
Asynchronous mid-infrared broadband optical parametric oscillator for dual-comb spectroscopy
}

\author{
Zhaowei Zhang, ${ }^{1}$ Chenglin Gu, ${ }^{\mathbf{1 , 2}}$ Jinghua Sun, ${ }^{1,4}$ Chingyue Wang, ${ }^{2}$ Tom Gardiner ${ }^{3}$ and Derryck T. \\ Reid $^{1}$ \\ ${ }^{1}$ Scottish Universities Physics Alliance (SUPA), Institute of Photonics and Quantum Sciences, \\ School of Engineering and Physical Sciences, Heriot-Watt University, Riccarton, Edinburgh EH14 \\ 4AS, UK \\ ${ }^{2}$ School of Precision Instruments and Optoelectronics Engineering, Tianjin University, Tianjin \\ 300072, China \\ ${ }^{3}$ National Physical Laboratory, Hampton Road, Teddington, London TW11 0LW, UK \\ ${ }^{4}$ School of Physics, Huazhong University of Science and Technology, 1037, Luoyu Road, Wuhan, \\ 430074, China
}

\begin{abstract}
Two asynchronous, broadband $3.3-\mu \mathrm{m}$ pulse trains with a stabilized repetition-rate difference of up to $5-\mathrm{kHz}$ were generated from a single optical parametric oscillator. With additional carrier-envelope-offset stabilization, it could be applied to coherent dual-frequency-comb spectroscopy.
\end{abstract}

\section{Introduction}

Dual-laser pulse trains with slightly different repetition rates are needed for asynchronous optical sampling (AOS) and dual frequency-comb spectroscopy (DFCS) [1-4]. The generation of asynchronous pulse trains normally begins with two nearly identical modelocked lasers, but convenient modelocked laser sources are not available in the mid-infrared (mid-IR), which is one of the spectral regions of most interest for DFCS. Optical parametric oscillators (OPOs) synchronouslypumped by near-IR modelocked lasers can satisfy this requirement by extending the spectral coverage to more than $6 \mu \mathrm{m}$.

In recent work we reported a synchronously-pumped OPO (SPOPO) producing broadband midIR pulses from a cavity containing a long MgO:PPLN crystal [5]. The high intracavity dispersion of this OPO made it insensitive to cavity-length variations, giving oscillation over a $120-\mu \mathrm{m}$ range (equivalent to an $8-\mathrm{kHz}$ repetition-rate detuning) with only $3 \mathrm{~nm}$ of signal tuning. This insensitivity can be exploited to allow the OPO to be pumped simultaneously by two lasers with different repetition rates, enabling asynchronous signal / idler pulse trains to be generated with only with slightly different central wavelengths. Here, we report the first, to our knowledge, SPOPO pumped by a pair of asynchronous pulse trains.

\section{Experiment and results}

The configuration of this asynchronous SPOPO is shown in Fig. 1. Two independent pump sources with identical structure and performance but slightly different repetition rates were combined by a

This is an Open Access article distributed under the terms of the Creative Commons Attribution License 2.0, which permits unrestricted use, distribution, and reproduction in any medium, provided the original work is properly cited. 
50:50 plate beam splitter. The average power, center wavelength, 3-dB spectral bandwidth and pulse width of each pump source were $2.5 \mathrm{~W}, 1058 \mathrm{~nm}, 10 \mathrm{~nm}(4.82 \mathrm{THz})$, and $\sim 3 \mathrm{ps}$ respectively. The repetition rate of pump Channel 1 was set at $100 \mathrm{MHz}$, while the repetition-rate difference, $\Delta \mathrm{f}$, between the two channels was stabilized to a reference frequency $\Delta_{\text {ref }}$ [6]. The OPO gain medium was a $20-\mathrm{mm}$ long and $1-\mathrm{mm}$ thick $5-\mathrm{mol} \% \mathrm{MgO}:$ PLN crystal with a grating period of $30.49 \mu \mathrm{m}$. The OPO was singly resonant for the signal, which was extracted through a $10 \%$ output coupler (OC). The generated idler passed through M2 and a Ge window isolated the idler pulses from the depleted pump.

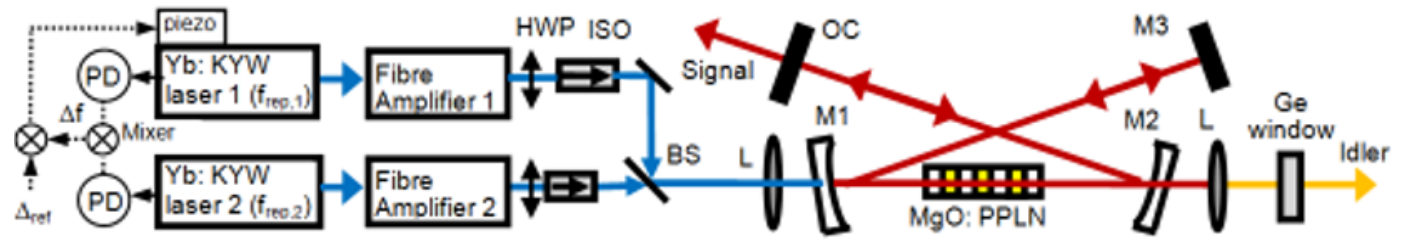

Fig. 1. The asynchronous SPOPO layout. HWP, half-wave plate; ISO, isolator; BS, beamsplitter; L, lens; PD, photodiode.

Figure 2 shows the measured signal / idler powers and spectra when the OPO was pumped individually by Channel 1 or Channel 2, or simultaneously by both channels, whose repetition-rate difference was stabilized to $20 \mathrm{~Hz}$. It is clear that when the OPO was pumped individually by Channel 1 or Channel 2, the OPO showed almost identical performance. Normally when the repetition frequency of the pulses pumping an SPOPO is changed the signal will move to a new center wavelength, so that the cavity round-trip time of the signal pulses still equals the pump-pulse interval. However the high group-delay dispersion of our system meant that there was only a 0.1-nm shift of the signal center wavelength. When the OPO was pumped simultaneously by both channels, the overall signal/idler output power was measured as the pump power of Channel 1 was varied, keeping Channel 2 pumped at full power. As shown in Fig. 2 (a) and (b), the measured overall output power was equal to the linear sum of the output powers when individually pumped. Also shown in Fig. 2 (c) and (d) are the signal and idler spectra when the OPO was pumped simultaneously by both channels at full pump power. These spectra are also nearly identical to the linear sum of the spectra obtained under individual pumping.
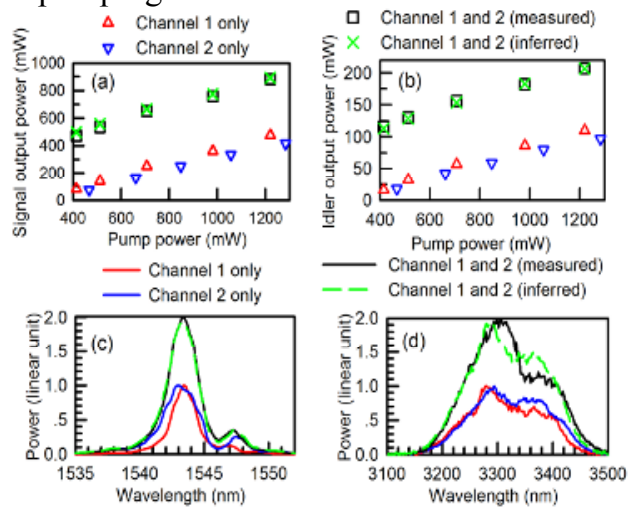

Fig. 2. (a) Signal and (b) idler output power. (c) Signal (d) idler output spectra $(\Delta \mathrm{f}=20 \mathrm{~Hz})$

The fact that when simultaneously pumped the OPO output is simply a linear summation of the outputs obtained when individually pumped shows that there is no significant nonlinear coupling between the two OPO channels. Further evidence confirming this is shown in Fig. 3, which is the direct measurement of the signal pulse trains of the OPO output. Due to the repetition rate difference of $1 \mathrm{kHz}$, the two signal pulse trains coincided every $1 \mathrm{~ms}$. Shown in Fig. 3(c) and (d) are, respectively, the combined signals when the two pulse trains overlap and do not overlap in the time domain. In both cases, the overall signal output is a linear summation of the two individual signal 
pulse trains. Therefore, each channel oscillates independently of the other, and the OPO with two pumping channels runs like two independent OPOs. This result is due to the instantaneous nature of the parametric gain process.
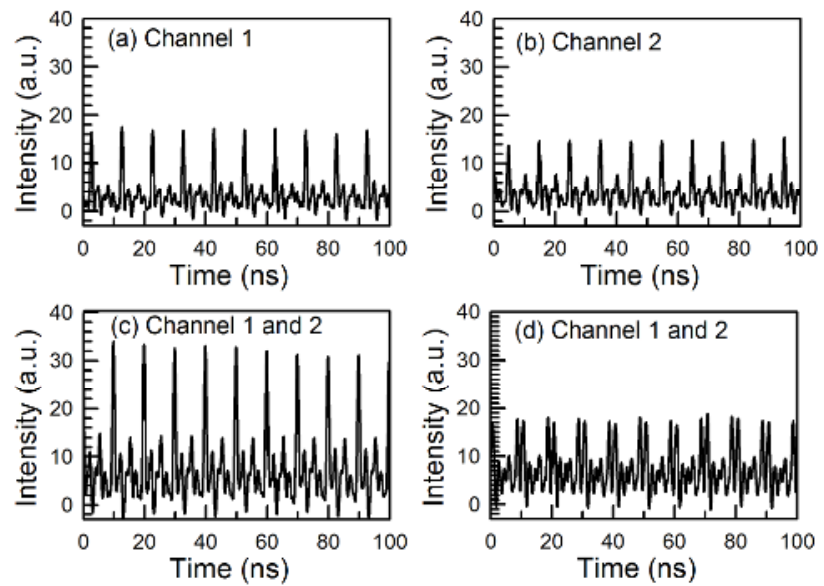

Fig. 3 The signal pulses at $\Delta \mathrm{f}=1 \mathrm{kHz}$ from (a) Channel 1 and (b) Channel 2. Channels 1 and 2 are shown simultaneously when overlapped in time in (c) and in (d) when not overlapped in time.

For an SPOPO with a fixed cavity length, the signal center wavelength can move automatically to adapt to a small change in the pump repetition rate, however phase-matching considerations mean that the signal tuning is confined to within a finite gain bandwidth. The signal output power falls if its center wavelength shifts away from the peak of this gain curve. Our experimental data show that, at a repetition rate difference of $\sim 5 \mathrm{kHz}$, the total signal output power only fell to $50 \%$ of the maximum. Even at $10-\mathrm{kHz}$ detuning, the offset in the signal central wavelengths of the two channels was below $1 \mathrm{~nm}$, implying only a 4-nm difference in the center wavelengths of the corresponding idler pulses. Further experimental results and details will be reported in the conference.

\section{Summary}

In summary, we have demonstrated a single OPO simultaneously pumped by two pump lasers which generates stabilized asynchronous mid-IR pulse trains with nearly identical spectral properties. The system has immediate applications in incoherent AOS and the low noise of the OPO [5] should make it well suited for this application. Coherent DFCS requires the carrier-envelope offsets of the idler pulses to be stabilized, and this can be readily achieved by heterodyning a pump supercontinuum with the pump-idler sum-frequency mixing light [8].

\section{References}

1. J. D. Kafka, J. W. Pieterse, and M. L. Watts, " Opt. Lett. 17, 1286-1288 (1992)

2. A. Bartels, R. Cerna, C. Kistner, A. Thoma, F. Hudert, C. Janke, and T. Dekorsy, Rev. Sci. Instrum. 78, 035107 (2007)

3. A. Schliesser, M. Brehm, F. Keilmann, and D. van der Weide, Opt. Express 13, 9029-9038 (2005)

4. I. Coddington, W. C. Swann, and N. R. Newbury, Phys. Rev. Lett. 100, 013902 (2008)

5. Z. Zhang, J. Sun, T. Gardiner, and D. T. Reid, Opt. Express 19, 17127 (2011)

6. Z. Zhang, T. Gardiner and D. T. Reid, Electron. Lett. 47, 1140-1142 (2011)

7. D. T. Reid, Z. Penman, M. Ebrahimzadeh, W. Sibbett, H. Karlsson, and F. Laurell, Opt. Lett. 22, 1397$1399(1997)$

8. J. H. Sun, B. J. S. Gale, and D. T. Reid, Opt. Lett. 32, 1414 (2007) 